\title{
A Comparison Study Between an Adaptive Quadtree Grid and Uniform Grid Upscaling for Reservoir Simulation
}

\author{
Masoud Babaei · Ahmed H. Elsheikh • Peter R. King
}

Received: 22 July 2012 / Accepted: 27 February 2013 / Published online: 13 March 2013

C Springer Science+Business Media Dordrecht 2013

\begin{abstract}
An adaptive quadtree grid generation algorithm is developed and applied for tracer and multiphase flow in channelized heterogeneous porous media. Adaptivity was guided using two different approaches. In the first approach, wavelet transformation was used to generate a refinement field based on permeability variations. The second approach uses flow information based on the solution of an initial-time fine-scale problem. The resulting grids were compared with uniform grid upscaling. For uniform upscaling, two commonly applied methods were used: renormalization upscaling and local-global upscaling. The velocities obtained by adaptive grid and uniformly upscaled grids, were downscaled. This procedure allows us to separate the upscaling errors, on adaptive and uniform grids, from the numerical dispersion errors resulting from solving the saturation equation on a coarse grid. The simulation results obtained by solving on flow-based adaptive quadtree grids for the case of a single phase flow show reasonable agreement with more computationally demanding fine-scale models and local-global upscaled models. For the multiphase case, the agreement is less evident, especially in piston-like displacement cases with sharp frontal movement. In such cases a non-iterative transmissibility upscaling procedure for adaptive grid is shown to significantly reduce the errors and make the adaptive grid comparable to iterative local-global upscaling. Furthermore, existence of barriers in a porous medium complicates both upscaling and grid adaptivity. This issue is addressed by adapting the grid using a combination of flow information and a permeability based heuristic criterion.
\end{abstract}

Keywords Adaptive quadtree grid - Permeability- and flow-based gridding · Renormalization upscaling $\cdot$ Local-global upscaling $\cdot$ Downscaling

M. Babaei ( $\varangle)$. A. H. Elsheikh · P. R. King

Department of Earth Science \& Engineering, Imperial College London, Prince Consort Road, London SW7 2BP, UK

e-mail: masoud.babaei08@imperial.ac.uk 


\section{Introduction}

The combined use of adaptive gridding and upscaling of reservoir properties is an active research area. Adaptive gridding can be thought of as a grid coarsening problem, where grid blocks are merged without compromising the simulation accuracy. Also, it can be thought of as a grid refinement problem where additional grid resolution is locally added throughout the simulation domain to increase the solution quality. In reservoir engineering, the grid coarsening view is usually adopted and two main sources of errors can be introduced during this process. Upscaling errors resulting from mapping fine scale properties to a coarser grid and the standard discretization errors resulting from using fewer degrees of freedoms to represent the solution. The impact of a uniform upscaling for high flow regions appears in the form of errors in the coarse scale velocity field and incorrect saturation profiles. As a result of that, unreliable predictions (e.g., exaggerated sweep and inaccurate breakthrough times) are often observed from uniform upscaling of highly heterogeneous reservoirs.

Upscaling errors can be addressed by using an adaptive gridding framework. Within such a framework, the mesh resolution is adjusted adaptively for physical processes that occur at a wide range of spatial and temporal scales. Adaptive gridding can be classified into adaptive Cartesian gridding and unstructured gridding. Adaptive Cartesian grids are constructed by patch-based refinement or by merging a collection of fine scale Cartesian cells. In unstructured gridding, the coarse grid blocks can be misaligned with the fine grid so that flow features can be captured more efficiently. Such flexibility can also be achieved by constructing structured curvilinear or elliptic grids.

Different criteria can be used for adapting the grid. For example, geometrical or geological information of reservoirs such as high permeability areas, fractures, faults, or boundaries can be used. Also, the grid can be adapted based on information obtained by the solution of a flow problem. This presents a possibility where grids can be constructed based on streamlines where areas with high density of streamlines are candidates for refinement. Furthermore, the grid can be aligned with streamlines to capture the flow paths efficiently.

Examples of the permeability-based gridding approach are Farmer et al. (1991), Li et al. (1995) and Qi et al. (2001). Generally, the heterogeneous areas of the domain were left at the finer scales and the homogeneous regions were coarsened. The spatial connectivity of a global permeability field were considered by Qi et al. (2001). The authors refined a grid block if the permeability variance is higher than a pre-specified threshold. Ebrahimi and Sahimi (2002) illustrated a high level of coarsening of fine geocellular models by utilizing wavelets to capture the heterogamous parts of the porous medium. Garcia et al. (1992) used geometric information of the initial permeability map to adjust the corners of a Cartesian mesh into curvilinear grids so that the variance of permeability within coarse grid blocks is minimized.

In other approach based on permeability variation, Younis and Caers (2002) used a "leaky" connected-set approach that delineates clusters of ne cells with similar permeability. Heuristic measures are used to colour each connected set, indicating the level of the importance of capturing the set by refinement. Refinement flags are then obtained by explicit boundary detection (Gerritsen and Lambers 2008).

Examples of flow- and streamline-based gridding are Durlofsky et al. (1996, 1997), Darman et al. (2001), Castellini et al. (2000), Wen et al. (2003) and Prevost et al. (2005). Mlacnik et al. (2006) constructed PEBI grids based on flow solution. Similarly, Edwards (2002) proposed construction of flow-based triangular grid. Moreover, Gerritsen and Lambers (2008) used leaky connected-set approach for constructing a "base grid" based on permeability variations, with further refinements made later according to flow by a multilevel local-global 
upscaling. Flow-based gridding or streamline-based gridding requires the solution of a fine scale problem. It is assumed that flow field will not undergo severe changes in later times of the simulation. The requirement of initial fine scale solution imposes a fairly small computational cost relative to the simulation of the multi-phase system (Durlofsky 2005).

Both criteria of flow and permeability variations can be combined for adapting the grid. Wen and Gómez-Hernández (1996) introduced an iterative procedure to add velocity variations to the grid constructed by Garcia et al. (1992). Elliptic Jacobian-based gridding was also proposed by He (2005) and He and Durlofsky (2006). It involves deriving the solution of a set of nonlinear elliptic equations and obtaining a transformation operator to map from physical to logical Cartesian space. A grid was constructed using both the obtained map and streamline information. This method is fast and simple to apply. However, in some cases, the convergence is slow and there is no guarantee the grid will have non-intersecting coordinate lines (Durlofsky 2005).

Cartesian adaptive quadtree grid (AQG) generation for reservoir simulation is relatively easy to implement and requires much less bookkeeping in comparison to unstructured grids. Also, from the reservoir simulation standpoint, upscaling can be more straightforward on AQG than on an unstructured grid. Permeability or transmissibility can be upscaled within each coarse grid block just with changing the level of upscaling. Nonetheless, the main drawback of quadtree grid is the lack of flexibility in representing severe geometrical deformities of porous media compared to an unstructured grid. This might lead to a high number of cells around the heterogeneities.

In this paper, we perform a critical evaluation of two different methods for quadtree grids construction based on permeability field information and flow-based information. The solution on AQG is compared with uniform grid coarsening with standard upscaling and iterative local-global upscaling method (Chen and Durlofsky 2006a). This problem differs from standard mesh adaptivity methods where the mesh is refined and coarsened based a discretization error metric. Here, both the discretization errors and upscaling errors are present. The list of the methods used is: (1) uniform mesh coarsening with renormalization upscaling for permeability, (2) uniform mesh coarsening with adaptive local-global upscaling for transmissibility, (3) quadtree grid coarsening based on permeability field indicator (wavelets) with renormalization permeability upscaling, (4) quadtree grid coarsening based on permeability field indicator (wavelets) with extended-local transmissibility upscaling, (5) quadtree grid coarsening based on flow field indicator with renormalization permeability upscaling, and (6) quadtree grid coarsening based on flow field indicator with extended-local transmissibility upscaling. These different methods are numerically tested against heterogeneous geological models with large permeability variations. In the numerical simulations, coarse scale velocities were downscaled back to the original fine grid in order to keep the errors bound to the absolution permeability upscaling. This allows us to solve the phase saturations at the fine grid for all the methods under consideration.

In this study we try to determine what is the best way to utilize mesh adaptivity via quadtrees combined with an upscaling method for solving multi-phase flow problems in heterogeneous medium. In that pursuit, we quantify and compare the performance of adaptive quadtree grids to the fairly accurate adaptive local-global upscaling algorithm.

Outline This paper includes the following sections. In Sect. 2 we will briefly describe the governing equations of flow in porous media and in Sect. 3 we review common upscaling methods. Next in Sect. 4 we will explain the construction of adaptive quadtrees and present the two different methods used to define the adaptivity criteria. In Sect. 5 we report the results of several numerical experiments. Finally, we end the paper with conclusions in Sect. 6. 


\section{Governing Equations}

The multiphase immiscible flow problem in porous media is described by mass conservation equation and Darcy's law:

$$
\nabla \cdot \mathbf{v}_{t}=q, \quad \mathbf{v}_{t}=-\mathrm{K} \lambda_{t} \nabla p, \quad \text { in } \Omega,
$$

where $q$ represents the total source and sink contributions, $\mathbf{v}_{t}$ is the total Darcy velocity of the engaging fluids, $\mathrm{K}$ is the tensor of absolute permeability, $\lambda_{t}$ is the total mobility, $p$ is the pressure and $\Omega$ the problem domain. These equations can be combined to produce the pressure equation $-\nabla \cdot\left[\mathrm{K} \lambda_{t} \nabla p\right]=q$. Gravity and capillary effects are neglected in the current formation.

In this article, we use finite difference method to discretize the pressure equation for both the uniform grid and quadtree grid. For the uniform grid a classical Two-Point Flux Approximation (TPFA) scheme is used. For the quadtree grid, an extra degree of care in needed to deal with truncation errors arising from non-aligned fluxes between two cells of unequal sizes (Quandalle and Besset 1985; Forsyth and Sammon 1986). A consistent discretization method proposed by Edwards (1996) is utilized. This scheme uses an extended stencil around the interface derived by flux and pressure continuity. It also produces a symmetric, positive definite and diagonally dominant matrix of coefficients.

After solving for the pressure and extracting fluxes between interfaces, the transport of phase $i$ can be formulated as:

$$
\phi \frac{\partial S_{i}}{\partial t}+\nabla \cdot\left[\mathbf{v}_{t} f_{i}\left(S_{i}\right)\right]=q_{i}, \quad \text { in } \Omega,
$$

where $\phi$ is porosity, $S_{i}$ is the saturation of phase $i, f_{i}\left(S_{i}\right)$ is the fractional flow of phase $i$ and $q_{i}$ is the source and sink term of phase $i$. The fractional flow of phase $i$ is defined by $f_{i}\left(S_{i}\right)=\lambda_{i} / \lambda_{t}$ where $\lambda_{i}=k_{\mathrm{r} i} / \mu_{i}$ is the mobility of phase $i, \lambda_{t}=\sum_{i} \lambda_{i}$ is the total mobility, $k_{\mathrm{r} i}$ is the relative permeability of phase $i$ and $\mu_{i}$ is the viscosity of phase $i$.

\section{Pressure Equation Upscaling}

One of the most common methods for upscaling the pressure equation is to calculate a tensorial equivalent permeability by solving a local problem. The local problem is defined around the region that surrounds the coarse grid block (see Durlofsky 1991; Pickup et al. 1994). This method is known as Pressure Solver Method (PSM). In PSM, the pressure equation is solved and an equivalent permeability value is calculated based on the obtained solution. To solve this local equation we have to assume a set of boundary conditions a priori. The most general case is to assume a periodic boundary conditions. In directional PSM (Begg et al. 1989; Christie 1996), the cross flow is ignored and only diagonal entries of the tensor are calculated. This requires applying a constant pressure gradient in one direction and no flow over the other directions. Such pressure gradient produces a directional flow over the local problem. An equivalent permeability tensor, $\mathbf{k}^{*}$ can be estimated by relating the computed flow to the assumed directional pressure gradient. Similarly, the procedure is repeated for other spatial directions to estimate the diagonal entries of an equivalent permeability tensor.

\subsection{Renormalization Method (RM) Permeability Upscaling}

King (1989) provided a closed form formula for approximating the equivalent permeability tensor $\mathbf{k}^{*}$. The algorithm is limited to hierarchically coarsened Cartesian cells. That is to 
coarsen from $N \times N$ to $\frac{N}{2} \times \frac{N}{2} \ldots$ to $4 \times 4$ to $2 \times 2$ to a final single cell, where $N$ is the number of fine cells in each direction. The upscaled permeability fields obtained by RM are very close to directional PSM with the benefit of not having to solve any system of equations. However, the assumed boundary conditions in both PSM and RM might not be realistic and that might affect the upscaled solution quality.

\subsection{Adaptive Local-Global (ALG) Transmissibility Upscaling}

Chen and Durlofsky (2006a) suggested this method to iteratively estimate the correct boundary conditions of the local problems which was assumed constant in RM and directional PSM. The boundary conditions are updated by using the global coarse pressure values. The quality of the solutions obtained by the iterative method is better than non-iterative methods because iterative methods takes into account the effects of long range spreading heterogeneities of porous media on flow and upscaled values. Moreover, the transmissibility upscaling avoids the secondary error otherwise encountered in calculation of coarse transmissibilities based on upscaled permeabilities (Chen et al. 2003). The term adaptive here, not to be confused with adaptive gridding, refers to a version of local-global upscaling that is designed to adapt to different boundary conditions and flow scenarios. This is a version which is different from the method introduced by Chen et al. (2003) where the upscaled values were driven from alternating axis-oriented generic boundary conditions.

In the case of multiphase flow, further to absolute permeability upscaling, the existence of $\lambda_{t}(S)$ as a strong spatially varying function of saturation creates problems as the saturation changes during the simulation time. Therefore, one has to consider the mobility changes into the upscaling process. One common approach is to define dynamic pseudo-functions. However, pseudo-function method is computationally expensive and depends on the flow conditions (Aarnes and Efendiev 2006). In other words, pseudofunctions may only be valid for the exact conditions for which they were derived. We could avoid using pseudofunctions for multiphase flow problems if we assume similar relative permeability relationships between the coarse and fine scale grids. This is justified, as we will explain later, if we solve the saturation equation at the fine scale with the relative permeabilities unchanged from the original mode.

In an attempt to upscale transmissibilities for an adaptive grid, Nilsson et al. (2005) and Gerritsen and Lambers (2008) integrated the transmissibility upscaling of local-global method with adaptive Cartesian anisotropic grid in two dimensions. They allowed refinement to be different in $x$ and $y$ directions so that the coarse grid is allowed to be rectangular. The results show an accurate upscaling for adaptive grid.

In this study, we use a simple transmissibility upscaling for adaptive grid. The procedure is based on assuming an extended region around the target edge of a given grid cell. Then, by imposing a directional pressure gradient and solving a local problem defined over the extended region, we can calculate the upscaled transmissibility. We do not perform iteration to correct the boundary conditions and the transmissibilities, however, for multiphase flow case we update the transmissibilities to account for the total mobility change throughout the simulation. In Fig. 1 the extended regions for two grid cells of different size are shown for the $x$ and $y$ directions.

\section{Adaptive Quadtree Grid (AQG) Generation}

A quadtree grid consists of a set of non-uniform Cartesian cells obtained from recursive subdivision of a quadrilateral cell called the root cell (de Berg et al. 2000). Each cell in the quadtree corresponds to a quadrilateral element and has four children and one parent. The 
Fig. 1 Transmissibility upscaling for adaptive quadtree grid. The hatched rectangles show two different-size grid cells. The segments $a b$ and $c d$ show two edges that we targeted for calculating the upscaled transmissibilities. The extended regions for these edges are, respectively, the blue and the green rectangles. Also shown are the boundary conditions imposed on these regions

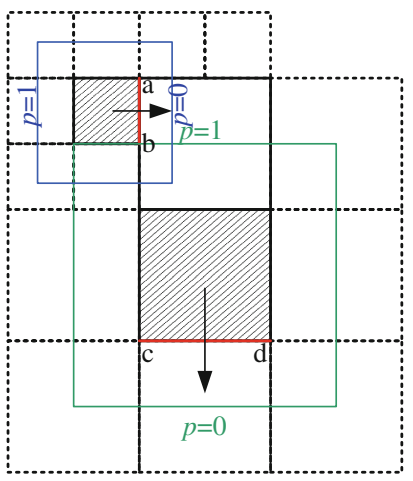

exception is the root cell which has no parent and the leaves which have no children. Dividing a leaf cell into four is denoted as a refinement operation and merging four children cells into their parent is denoted as a coarsening operation. In the current work, each quadtree cell is assessed based on a certain metric and if its value exceeds a minimum threshold, that cell is marked for refinement. Different refinement criteria can be used within the same adaptivity algorithm. Following the marking step, a grid regularization operation is performed to ensure that no cell has a side length more than twice the size of its neighbours. This step is called tree balancing. After finishing the marking and balancing phases, the actual refinement is done by dividing each cell into four children and updating the neighbouring relationship between the different cells.

In the context of subsurface flow modelling, the criteria for generating optimal quadtrees are not always entirely clear. Consequently, we will investigate two different strategies for marking quadtree cells for refinement; permeability- and flow-based criteria. In the following subsections we present a detailed description for each of these criteria.

\subsection{Wavelet Transformation for Permeability-Based Gridding}

Wavelets are used here for the permeability-based gridding. Wavelets have been applied successfully for grid generation on cases of high heterogeneity by Ebrahimi and Sahimi (2002, 2004) and Rasaei and Sahimi (2008a,b). The main idea is to filter the detail (or wavelet) coefficients obtained by transformation to reduce the size of data set or to eliminate fluctuations. During the thresholding, a wavelet coefficient is compared with a specified value and is set to zero if its magnitude is less than the threshold; otherwise, it is retained or modified depending on the thresholding rule. VisuShrink and SureShrink are two commonly used wavelet thresholding rules. For a signal with size $n$, VisuShrink which is also referred to as universal threshold (Donoho 1995) is defined as:

$$
t=\sigma \sqrt{2 \log n}
$$

where $\sigma$ is the noise level or equivalently $\sigma^{2}$ is the noise variance present in the signal. For practical use, when the noise level cannot be assumed to be known, it is important to estimate it. Based on the median absolute deviation, Donoho and Johnstone (1995) defined an estimate of the noise level as:

$$
\hat{\sigma}=\frac{\operatorname{median}\left(d_{j, k}\right)}{0.6745}
$$


where $d_{j, k}$ corresponds to the detail coefficients in the wavelet transform at scale $j$ and position $k$. The value 0.6745 is used for normalization because the median absolute deviation of the detail coefficients converges to this value times the noise level as $n$ goes to infinity. VisuShrink yields an overly smoothed fields as it applies a global thresholding scheme where a single threshold value is applied globally to all the wavelet coefficients.

Alternatively, SureShrink (Donoho and Johnstone 1995) is an adaptive threshold estimator based on the Stein's Unbiased Risk Estimator (SURE) (Stein 1981). It combines the universal threshold and the SURE threshold estimator and the goal is to minimize the mean squared error. The full mathematical details of the SureShrink estimator can be found in Donoho and Johnstone (1995). The thresholding employed by SureShrink will be adaptive and level dependent, that is, a different threshold value is assigned to each resolution level in the wavelet transform. This technique is also smoothness adaptive, which means that the reconstructed image preserves the abrupt changes or boundaries in the original image.

The SureShrink thresholding is applied here on wavelet transformation of permeability field by utilizing MATLAB built-in rigrsure option for threshold selection. The reconstruction of an inverse transform yields a map with similar size of the original field. If any variation is detected in the reconstructed permeability map (filtered) within a coarse grid cell, that cell is marked for refinement.

Here we note that, in using the permeability variation and wavelet transformation to create adaptive Cartesian gridding, we have not introduced a novelty, as Rasaei and Sahimi (2008b) used wavelet transformation of permeability field for the same purpose. However, the difference is that, they implemented two thresholds, $\epsilon_{s}$ and $\epsilon_{d}$, similar to Ebrahimi and Sahimi (2002). These thresholds are set as a fraction of their corresponding largest values throughout the grid. The scale coefficient of each block is then examined. If it is larger than $\epsilon_{s}$ (implying that the blocks permeability is large), it is left intact, and the next grid block is examined. If, however, the examined scale coefficient is smaller than $\epsilon_{s}$, the associated detail coefficients are examined and set to zero if they are smaller than their threshold $\epsilon_{d}$. Instead of using these thresholding we use SureShrink threshold which is, to our knowledge, new and more automated.

\subsection{Flow-Based Adaptive Gridding}

For flow-based adaptivity, the refinement/coarsening indicator is based on the maximum velocity magnitude within a coarse grid. The indicator for a cell $E$ is defined as:

$$
I_{E}=\max _{e \in E}\left\{\mathbf{v}_{e}\right\}
$$

where $\mathbf{v}_{e}$ is the cell-centred velocity magnitude at a subgrid cell $e$ inside the coarse grid cell $E$. The velocity magnitude is defined as

$$
\mathbf{v}_{e}=\sqrt{\mathbf{v}_{x}^{2}+\mathbf{v}_{y}^{2}}
$$

where $\mathbf{v}_{x}$ and $\mathbf{v}_{y}$ are the cell-centred averaged velocity values in the $x$ and $y$ directions, respectively. The velocity values are obtained from a single-stage calculation of fine scale velocity from solution of pressure equation with the boundary conditions of the simulation. For example the boundary condition can be one quarter of five spot pattern or constant pressure gradient.

The quadtree leaves are marked for refinement based on the relative value of $I$. In doing so, a user-defined percentile of the values of $I$ is determined. Then all the leaf cells that have an indicator value exceeding the percentile value are marked for refinement. The velocity 
field is obtained by solving the fine scale problem at the initial time step. The use of percentile automates the refinement for different heterogeneous media.

Here we note that, as we mentioned in the introduction, the criterion of flow for adapting the grid has been extensively used in literature. For example, in context of reservoir engineering and for Cartesian adaptive grid generation, Gerritsen and Lambers (2008) used a refinement indicator that is a function of a heuristically specified proportionality constant that depends on the ratio of areas between cells and a term that can be interpreted as the face's "fair share" of the global flow. Although this indicator and ours both similarly need global flow, their formulation are different and may lead to different meshes. Mainly, the indicator by Gerritsen and Lambers (2008) is based on fluxes across the faces, whereas we processed the fluxes to obtain cell-centred magnitudes of velocity. Moreover we will not use permeability-based grid for flow-based grid unless otherwise stated. Nonetheless, to decide which indicator results in higher quality mesh, more accurate prediction of flow, more aggressive grid adaptation or more computational efficiency, only direct comparison via numerical experiments must be employed. This, unfortunately, is currently beyond our ability.

We note that using different global boundary conditions to generate flow-based adaptive grid results in different meshes. For example, in this work, we use a corner-to-corner flow, that may result in more high velocity regions than the actual simulation, or quite in opposite, may not cover high flow regions induced by other cases of boundary conditions. For such circumstances, we refer to the same implications which were discussed in flow-based nonuniform coarsening presented in Durlofsky et al. (1997). The authors used the pressure-flux boundary condition which sets a constant pressure gradient in one direction and no flow on the sides of the other direction. With the aforementioned implications, the authors argued that an over-determination of high flow regions is preferred to an under-determination. Furthermore, the cost of resolving for missed high flow regions is not significant either.

\subsection{A Step-by-Step Algorithm for AQG Generation}

1. If using wavelets, map the permeability with wavelet transformation by a filtering procedure. However, one can use wavelet discrete transform functions developed under any other programming languages for the same filtering purpose.

2. If using flow-based gridding, first use the fine scale permeability and the specified boundary conditions, calculate the fine scale velocity field and the cell-centred magnitude of velocity based on Eq. 6.

3. Starting with the current grid (initially uniform $8 \times 32$ ), use either the filtered map of permeability or the magnitude of velocity for the refinement marking step.

The refinement for the wavelet-based quadtree grid is based on detection of variation in permeability of the constituent cells of a targeted coarse grid block because for the homogeneous coarse grid blocks, the constituent cells all have the same filtered permeability value.

The refinement for the flow-based quadtree grid is based on ranking of the coarse grids based on magnitude of velocity. That is, first we calculate, for each coarse grid, maximum value of the magnitude of velocity of the constituent fine cells. Then we calculate a specified percentile of the set of maximum values of the magnitude of velocity for the coarse grid blocks. Here, we decide that the coarse grid blocks with maximum value of the magnitude of velocity higher than 70th percentile are assumed to belong to high flow region and consequently are marked for refinement. We note that we also could have decided the coarse grids with maximum value of the magnitude of velocity lower than 

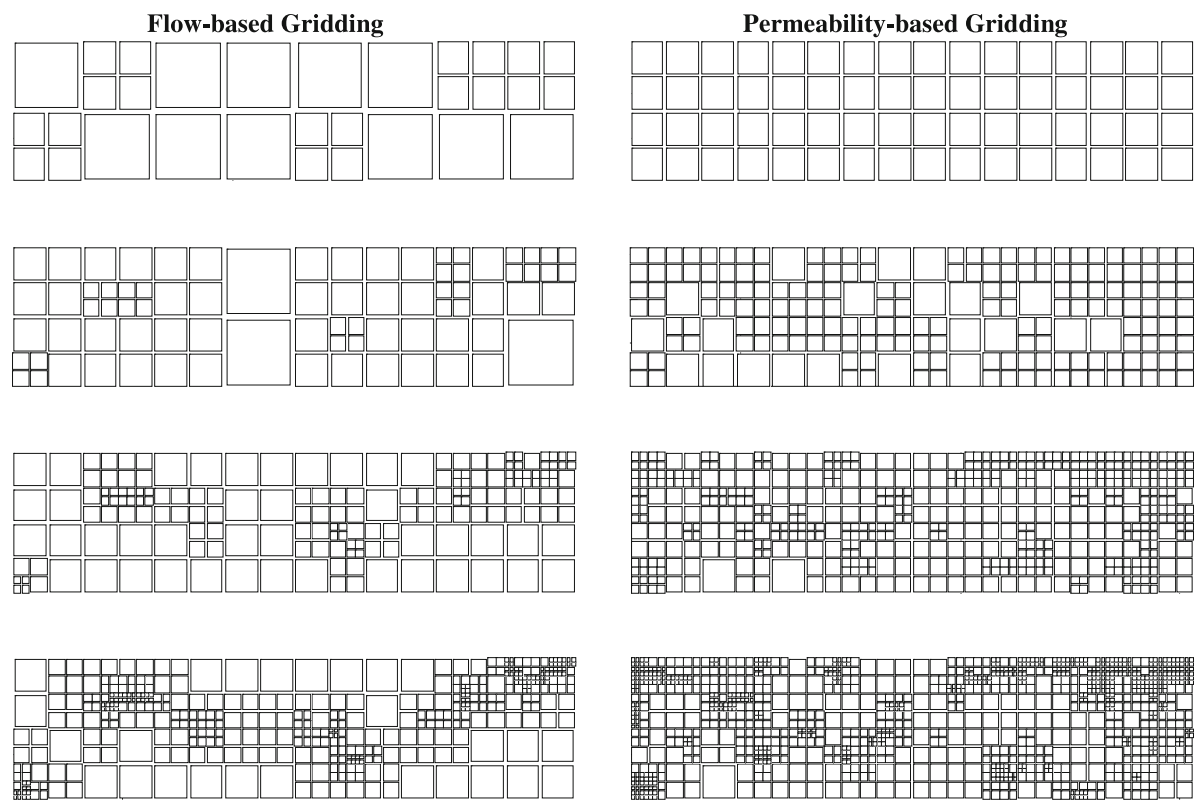

Fig. 2 Step by step construction of flow- and permeability-based grids for layer 37 of SPE10 model from an $8 \times 32$ uniform coarse grid (not shown) to the last adaptive quadtree grid. We imposed the layer with two wells at the lower left and upper right corners. We note that for the permeability-based gridding, we added the position of wells to the criteria of refinements at lower left and upper right corners

30th percentile be marked for coarsening, however, we did not implement this coarsening stage.

Also we do not allow the neighbouring cells to be refined so that the level of the refinement between them would be more than one. Therefore extra grids are marked for refinement. Moreover, we do not allow refinement exceeds a prescribed maximum level of refinement.

4. Perform the refinements of the marked grid blocks. Each coarse grid block is refined to four smaller grids.

5. Now we have a non-uniform grid with new number of cells. We perform stage 3 (with new refinement marking values from the new grid) until we finish the loop for the maximum number of refinement (see Fig. 2 for the repetition of stage 3 for the layer 37 of SPE10 model).

As a final remark about the construction of AQG grid, we note that the algorithm of refinement here is a function of two parameters: "maximum level of refinement" and "number of refinement loops". The first parameter, decides how fine the resolution of the refinement area can be, while the second allows us to choose the spatial extent and span of the refinement area. In the numerical experiments we specify a fixed maximum level of refinement and two consecutive values for the number of refinement loops that lead to two models of permeabilityand flow-based quadtree grids. An increase in the accuracy of each model is possible if we increase the number of refinement iterations or the maximum refinement level. However, this increase comes at the expense of higher number of cells and the additional computational cost. 


\subsection{Fine Scale Velocity Reconstruction}

The pressure equation upscaling can be deemed as the first stage of a two-stage upscaling procedure, the second stage being the saturation equation upscaling. For this stage, unlike the pressure equation upscaling, the form of the upscaled equation can be different from the fine scale (Durlofsky 1997). This is largely due to effects of subgrid flow on this equation and the important role of relative permeability relationships that may vary from fine to coarse scale grids. Such considerations have led to development of certain upscaling techniques that only deal with the saturation equation (see Efendiev and Durlofsky 2003; Chen and Durlofsky 2006b; Chen and Li 2009). In the current study, we limit ourselves to pressure equation upscaling and solve the saturation equation on the fine scale grid.

In all our numerical testing we solve for the pressure distribution in the upscaled or adapted grid and include the fine scale heterogeneities in the computation of the phase saturations in the original fine grid. In other words we solve the saturation at a grid nested inside the coarse grid, (Nested Gridding simulation), or under Dual Mesh framework.

In this work we use a static mass-conservative velocity reconstruction method developed in Babaei and King (2012). The algorithm uses an initial-time solution of a set of unit-value Neumann boundary problems for each coarse grid. Each set comprises four problems, number of coarse edges in $2 \mathrm{D}$, which are stored and used for the rest of the simulation by a linear superposition with coarse scale velocity as weights. Here, for adaptive grids, we use a similar algorithm with minimal adaptation to account for coarse grid blocks with variable sizes.

The reconstructed fine scale velocity is then used to calculate the saturation of each phase at the fine scale. Although we do not use any procedure to save computation in this stage, the literature provides several techniques that are designed to use a fine scale velocity field as an input. These techniques can be easily applied here. Few examples are streamline method (Batycky et al. 1997) and adaptive multiscale transport solvers (Aarnes and Efendiev 2006; Lee et al. 2009).

\section{Numerical Test Cases}

In this section we analyse results of flow simulation using AQG algorithm on several layers extracted from Model 2 of the 10th SPE Comparative Solution Project (Christie and Blunt 2001), (henceforth referred to as the SPE10 model). The bottom 50 layers of SPE10 model, represent a fluvial formation with narrow high-flow channels which makes these models difficult to upscale. The layers originally consist of $60 \times 220$ fine cells. However, for the purpose of application of renormalization upscaling and quadtree gridding, we modified these layers to $64 \times 256$ cells layout. In order to separate the effect of multiphase flow upscaling from single phase flow upscaling, the flow function is considered as two cases: the tracer (linear) flow case and the multiphase (non-linear) flow case.

In tracer flow we assume that the relative permeabilities of engaging fluids (oil and water) are linear function of their saturations and their viscosities are equal. In other words, oil and water are identical fluids. This assumption leads to a constant total mobility value throughout the simulation, so that the upscaling problem only concerns absolute permeability and not multiphase relative permeabilities. The pressure field and consequently the velocity field need to be calculated only once at the initial time step. Hence, the evolution of tracer concentration does not affect the upscaled properties. For the multiphase case we assume relative permeabilities relationships that lead to varying total mobility values. We use a Corey-type (Corey 1954) relative permeability relationships for oil and water: 


$$
k_{r o}=\alpha_{1}\left(1-S_{\mathrm{wD}}\right)^{m}, \quad k_{\mathrm{rw}}=\alpha_{2}\left(S_{\mathrm{wD}}\right)^{n},
$$

where $\alpha_{1}=1.0, \alpha_{2}=1.0, m=2, n=2$ and $S_{\mathrm{wD}}=\left(S_{\mathrm{w}}-S_{\mathrm{wc}}\right) /\left(1-S_{\mathrm{or}}-S_{\mathrm{wc}}\right) . S_{\mathrm{w}}, S_{\mathrm{wc}}$ and $S_{\mathrm{or}}$ are water saturation, connate water saturation and residual oil saturation, respectively.

Two different viscosity ratios are assumed. Viscosity ratio of $M=\mu_{\mathrm{o}} / \mu_{\mathrm{w}}=10$ that creates an unstable front movement with smearing effects due to protrusion of less viscous displacing fluid through the displaced one and viscosity ratio of $M=\mu_{\mathrm{o}} / \mu_{\mathrm{w}}=0.1$ that induces a piston-like movement of displacing fluid with a very sharp front.

The simulations were performed on the following models: uniform coarse grid models (1024 cells: coarsening level 1 and 256 cells: coarsening level 2) with upscaled permeabilities obtained by renormalization method, or upscaled transmissibilities obtained by adaptive local-global upscaling method. We denote these models by RM 1024 cells, RM 256 cells, ALG 1024 cells and ALG 256 cells. Two models of permeability-based AQG (coarsening levels 1 and 2, obtained by different numbers of refinement loops), with either the permeabilities of coarse grids obtained by renormalization or transmissibilities of coarse edges by extended-local transmissibility upscaling. These models are denoted by $k$-AQGk* or $k$-AQGT* followed by the number of cells in each model for the renormalization-based upscaling and the edge-based transmissibility upscaling, respectively. Finally, two models of flow-based AQG, with again upscaled permeabilities or upscaled transmissibilities denoted by $q$-AQGk* or $q$-AQGT* followed by the number of cells in each model.

For all the models, the pressure equation is solved on a coarse grid and the velocity is downscaled by uniform-grid or adaptive-grid nested gridding procedure. The saturation is then calculated on the original fine scale grid. This assures that the upscaling errors are separated from numerical dispersion error arising from coarse representation of saturation. For boundary and well conditions we assume a source (constant injection flow rate) and a sink (constant bottom-hole pressure production), respectively, on the lower left and the upper right corners of the system. This configuration will induce a corner to corner flow. A no flow boundary condition is applied on the domain outer boundaries.

For the assessment of each model, a comparison to the fine scale reference solution is performed. The following error measures are considered:

1. The saturation error (Hauge et al. 2011):

$$
\delta(s)=\frac{1}{T} \int_{0}^{T} \frac{\left\|s(., t)-s_{\mathrm{ref}}(., t)\right\|_{2}}{\left\|s_{\mathrm{ref}}(., t)\right\|_{2}} \mathrm{~d} t,
$$

where $s$ is the saturation calculated from reconstructed velocity field of the model under consideration and $s_{\text {ref }}$ is the saturation from the fine scale reference model. Further, $t$ is time measured in pore-volumes injected (PVI) and $T$ is the final simulation time. Finally $\|\cdot\|_{2}$ represents Euclidean norm or $L^{2}$ norm. Obtaining $\delta(s)$ at $T$, we calculate a relative error for RM, $q$-AQG and $k$-AQG compared to ALG by

$$
\epsilon_{r}(s)=\frac{\delta(s)_{l} \times N_{l}}{\delta(s)_{\mathrm{ALG}} \times N_{\mathrm{ALG}}},
$$

where $l$ represents the models which could be: RM, $q$-AQGk*, $q$-AQGT*, $k$-AQGk* or $k$-AQGT* and $N$ is the number of cells in each model. Assuming that error reduces linearly with larger number of cells, this value represents an approximate estimation of accuracy of each method compared to ALG in terms of quality of the saturation profile. 
2. The reconstructed velocity error (Kippe et al. 2008):

$$
\delta(v)=\frac{\left\|v_{x}-v_{x}^{\mathrm{ref}}\right\|_{2}}{\left\|v_{x}^{\mathrm{ref}}\right\|_{2}}+\frac{\left\|v_{y}-v_{y}^{\mathrm{ref}}\right\|_{2}}{\left\|v_{y}^{\mathrm{ref}}\right\|_{2}},
$$

where $v_{x}$ and $v_{y}$ are reconstructed velocity components in $x$ and $y$ directions and $v^{\text {ref }}$ is the fine scale reference velocity field. This error illustrates the quality of reconstructed velocity and indirectly examines the accuracy of the coarse velocity of certain model used in the downscaling procedure. Similar to saturation, we calculate a relative error for $\mathrm{RM}, q-\mathrm{AQG}$ and $k$-AQG compared to ALG by

$$
\epsilon_{r}(v)=\frac{\delta(v)_{l} \times N_{l}}{\delta(v)_{\mathrm{ALG}} \times N_{\mathrm{ALG}}},
$$

where $l=\mathrm{RM}, q$-AQGk* $*$-AQGT*, $k$-AQGk* or $k$-AQGT* and $N$ is the number of cells in each model.

3. The water cut versus PVI obtained from each model. Water cut is measured by fraction of the water mobility divided by the total mobility. In case of constant total mobility this fraction is equal to the water saturation of producing cell. The value is at any case (tracer or multiphase flow) a function of the single cell's saturation. Clearly, these values are local in contrast to the previous global norms. Furthermore, we have to note that, for all the methods, the water cut values are obtained based on the fine scale saturation results.

\subsection{Tracer Flow Simulations}

Here, we perform simulations on smoothly heterogeneous layer 10 and two channelized layers, 37 and 47 of SPE10 model (Christie and Blunt 2001). Layer 10 shown in Fig. 3a is selected to investigate how different models perform at moderately heterogeneous medium. The constructed grids and saturation profiles are shown in Fig. 3. From wavelet filtered permeability map shown in Fig. 3c, we observe that regions of high permeability are not filtered out by the wavelet filtering. It is also observed that the fine scale velocity and the reconstructed velocities resulted in a similar saturation fields at $t=0.6 \mathrm{PVI}$. Further, the uniform grid upscaling and AQG models yielded almost identical water cut curves (shown in Fig. 4) that match the fine scale results. The match is complete in cases of ALG, $q$-AQGk* and $q$-AQGT* models and is reasonable for RM, $k$-AQGk* and $k$-AQGT* models. This assures that the downscaling effectively eliminated the dispersion error in all coarse and adaptive grid models.

Next, the channelized layers 37 and 47 are examined. The constructed grids and saturation profiles for layer 37 at $t=0.6 \mathrm{PVI}$ are shown in Fig. 5. Additionally, the saturation profiles obtained from downscaling of ALG and RM are shown for comparison. Continuing the simulations till $t=1.2 \mathrm{PVI}$, we obtain $\delta(s)$ and $\epsilon_{r}(s)$ which are reported in Table 1 for layer 37 and in Table 2 for layer 47. In the same tables we reported $\delta(v)$ and $\epsilon_{r}(v)$. From the tables we draw the following points.

Starting from the permeability upscaling AQG model, the two $k$-AQGk* models show minimal improvements in $\delta(s)$ and $\delta(v)$, if any, in comparison to uniform RM. Regarding the number of cells, $\epsilon_{r}(s)$ and $\epsilon_{r}(v)$ for $k$-AQGk* are considerably higher than those of RM, showing that addition of cells has not been effective at all. For $q$-AQGk*, coarsening level 2 , resulted in lower values of $\delta(s)$ and $\delta(v)$ compared to RM 256 cells. However, the degree of reduction is not emphatic and the values of $\epsilon_{r}(s)$ and $\epsilon_{r}(v)$ are higher than those of RM 256 cells. On the other hand, $q$-AQGk*, coarsening level 1, produced $\epsilon_{r}(s)$ and $\epsilon_{r}(v)$ that 


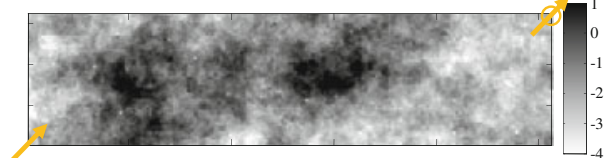

(a)

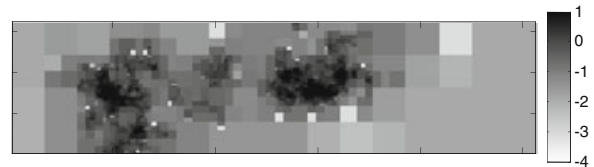

(c)

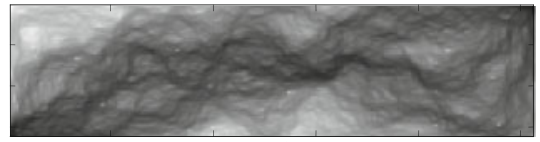

(e)

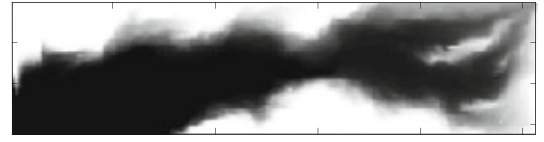

(g)

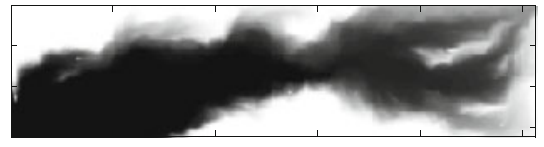

(i)

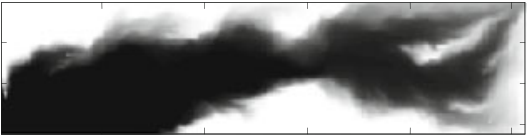

(b)

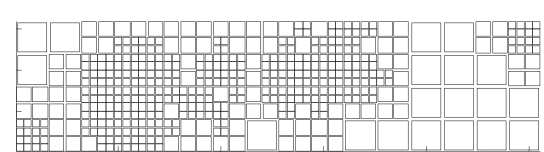

(d)

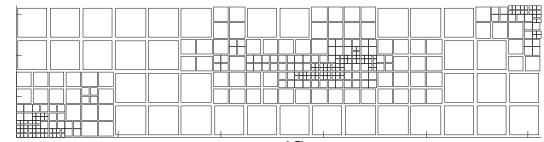

(f)

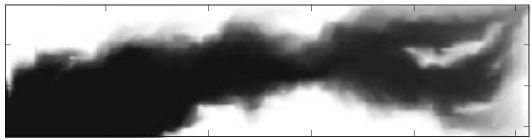

(h)

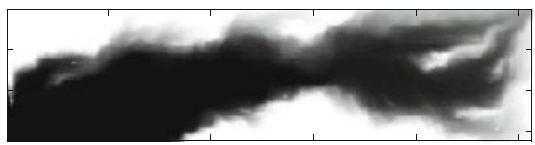

(j)

Fig. 3 Layer 10 tracer flow simulation. a Layer 10's original fine scale permeability; b fine scale saturation at $0.6 \mathrm{PVI}$; $\mathbf{c}$ the filtered permeability obtained by wavelet transformation; $\mathbf{d} k$-AQG model constructed based on $\mathbf{c} ; \mathbf{e}$ logarithm of cell-centred velocity magnitude; $\mathbf{f} q$-AQG model constructed based on $\mathbf{e} ; \mathbf{g}, \mathbf{h}, \mathbf{i}$, and $\mathbf{j}$ fine saturation solution at $0.6 \mathrm{PVI}$ obtained by use of velocity reconstructed from, respectively, $k$-AQGk*, $q$-AQGk* $k$-AQGT* and $q$-AQGT*

Fig. 4 Water cut curves obtained by different models for layer 10 tracer flow simulation

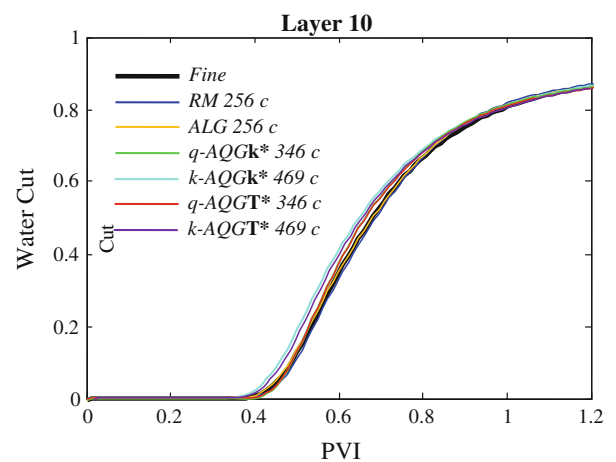

are around the same range of RM 1024 cells, with only 0.827 of the total number of cells for layer 37 and 0.625 of the total number of cells for layer 47. Consequently $\epsilon_{r}(s)$ and $\epsilon_{r}(v)$ for this model is lower than RM 1024 cells. 


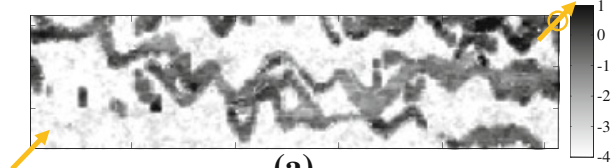

(a)

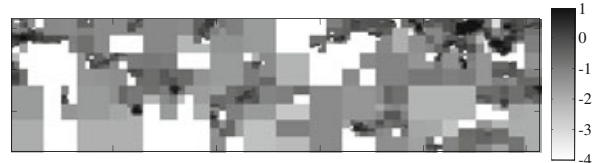

(c)

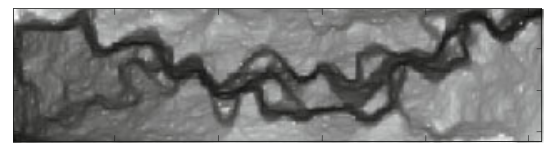

(e)

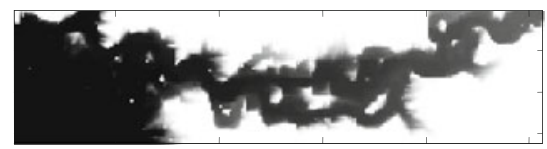

(g)

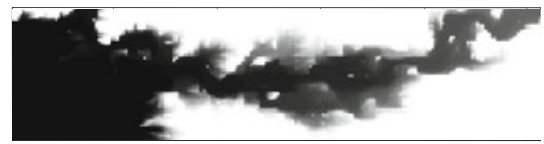

(i)

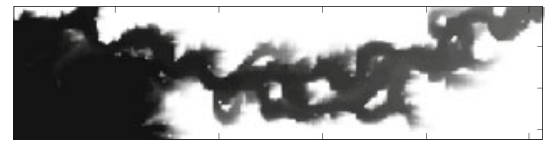

(k)

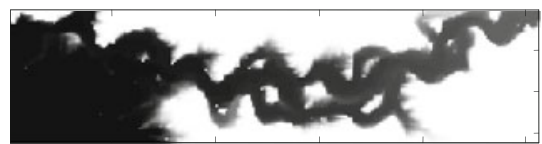

(b)

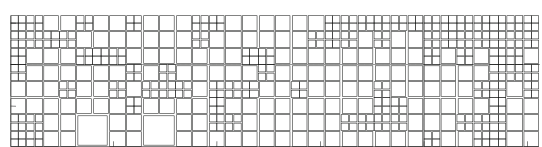

(d)

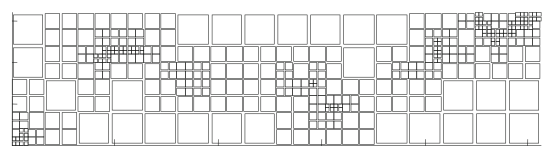

(f)

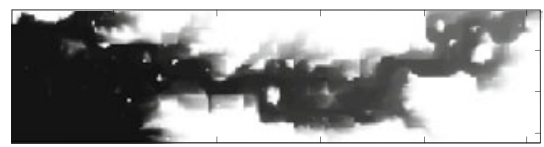

(h)

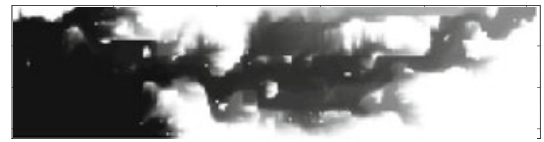

(j)

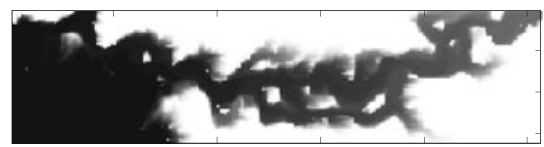

(1)

Fig. 5 Layer 37 tracer flow simulation. a Layer 37's original fine scale permeability; b fine scale saturation at $0.6 \mathrm{PVI}$; $\mathbf{c}$ the filtered permeability obtained by wavelet transformation; $\mathbf{d} k$-AQG model constructed based on $\mathbf{c} ; \mathbf{e}$ logarithm of cell-centered velocity magnitude; $\mathbf{f} q$-AQG model constructed based on $\mathbf{e} ; \mathbf{g}$ the saturation profile at 0.6 PVI from ALG; (h) the saturation profile at 0.6 PVI from RM; (i), (j), (k) and (l) fine saturation solution at $0.6 \mathrm{PVI}$ obtained by use of velocity reconstructed from, respectively, $q$-AQGk*, $k$-AQGk*, $q$ AQGT* and $k$-AQGT*

For the transmissibility upscaling AQG models, we observe considerable improvements in global error norms, even if this is a tracer flow case and the transmissibilities are only calculated at initial time. These improvements can be attributed to the extended local regions used for upscaling. Compared to ALG, $q$-AQGT*, coarsening level 1 , shows relative errors of around 0.8 and 1.4 suggesting close performance to ALG in terms of global errors for both saturation and velocity. Given that we do not implement any iteration, $q$-AQGT* can be rendered quite successful.

In terms of locally measured quantities, the water cut curves are depicted in Fig. 6 for layer 37 and Fig. 7 for layer 47 at two coarsening levels. $q$-AQGk* $q$-AQGT* and $k$-AQGT* are performing very similar to ALG. For $k$-AQGk* models, the curves are identical to RM curves and no improvement in the quality of the solution is observed by increasing the number of cells. 
Table 1 The saturation and velocity errors for layer 37 tracer flow simulation

\begin{tabular}{lllll}
\hline Model/Method & $\delta(s)$ & $\epsilon_{r}(s)$ & $\delta(v)$ & $\epsilon_{r}(v)$ \\
\hline RM 1024 c & 0.2237 & 2.5078 & 0.9636 & 3.0474 \\
RM 256 c & 0.3149 & 1.9669 & 1.1245 & 2.2101 \\
ALG 1024 c & 0.0892 & - & 0.3162 & - \\
ALG 256 c & 0.1601 & - & 0.5088 & - \\
$q$-AQGk* 847 c & 0.2213 & 2.0521 & 0.7517 & 1.9664 \\
$q$-AQGk* 403 c & 0.2927 & 2.8780 & 0.9467 & 2.9291 \\
$k$-AQGk* 1066 c & 0.2477 & 2.8908 & 0.9029 & 2.9726 \\
$k$-AQGk* 508 c & 0.2836 & 3.5151 & 1.0014 & 3.9056 \\
$q$-AQGT* 847 c & 0.1547 & 1.4345 & 0.4638 & 1.2133 \\
$q$-AQGT* 403 c & 0.1788 & 1.7581 & 0.6499 & 2.0108 \\
$k$-AQGT* 1066 c & 0.1865 & 2.1766 & 0.5412 & 1.7818 \\
$k$-AQGT* 508 c & 0.1958 & 2.4269 & 0.6445 & 2.5136 \\
\hline
\end{tabular}

Table 2 The saturation and velocity errors for layer 47 tracer flow simulation

\begin{tabular}{lllll}
\hline Model/Method & $\delta(s)$ & $\epsilon_{r}(s)$ & $\delta(v)$ & $\epsilon_{r}(v)$ \\
\hline RM 1024 c & 0.3227 & 3.1699 & 0.7828 & 2.6554 \\
RM 256 c & 0.4464 & 2.1861 & 1.2859 & 2.8531 \\
ALG 1024 c & 0.1018 & - & 0.2948 & - \\
ALG 256 c & 0.2042 & - & 0.4507 & - \\
$q$-AQGk* 640 c & 0.3775 & 2.3173 & 0.8074 & 1.7118 \\
$q$-AQGk*304 c & 0.4398 & 2.5578 & 0.9759 & 2.5713 \\
$k$-AQGk* 1363 c & 0.3794 & 4.9607 & 0.8494 & 3.8351 \\
$k$-AQGk* 583 c & 0.4221 & 4.7075 & 0.9673 & 4.8877 \\
$q$-AQGT* 640 c & 0.2152 & 1.3212 & 0.3849 & 0.8160 \\
$q$-AQGT* 304 c & 0.2356 & 1.3701 & 0.4160 & 1.0961 \\
$k$-AQGT* 1363 c & 0.1494 & 1.9534 & 0.4536 & 2.0481 \\
$k$-AQGT* 583 c & 0.2365 & 2.6376 & 0.6735 & 3.4031 \\
\hline
\end{tabular}
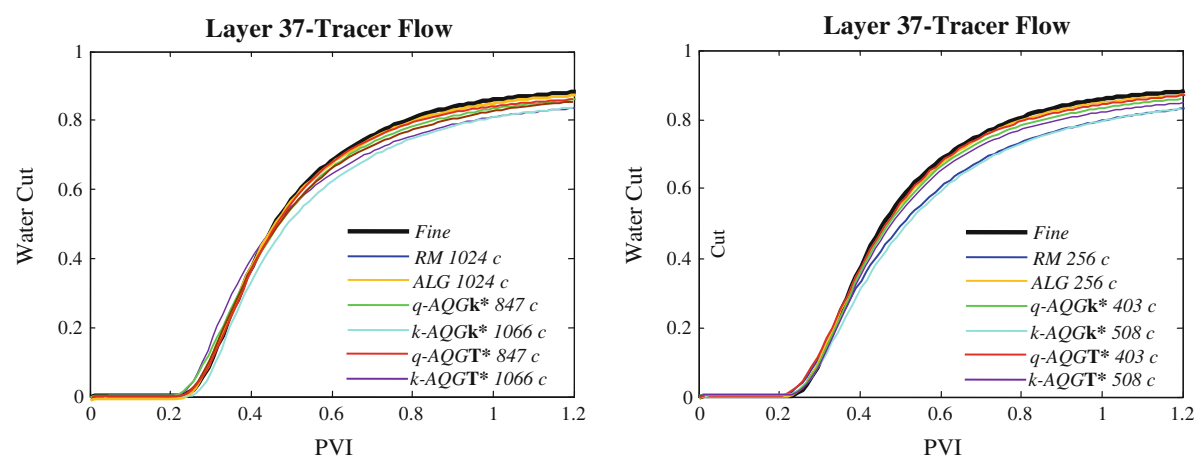

Fig. 6 Water cut curves obtained by different models for layer 37 tracer flow simulation at coarsening level 1 (left) and coarsening level 2 (right) 

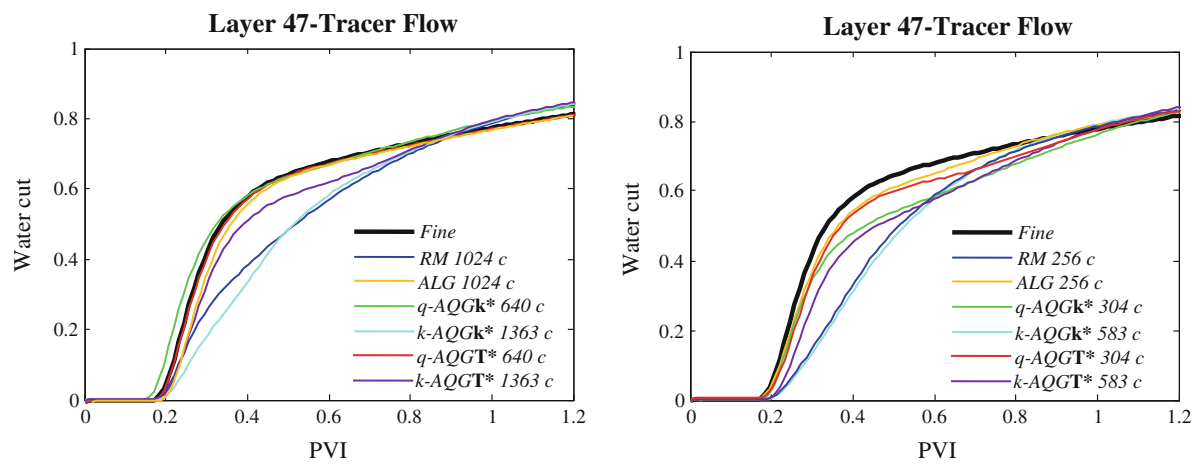

Fig. 7 Water cut curves obtained by different models for layer 47 tracer flow simulation at coarsening level 1 (left) and coarsening level 2 (right)

To this end, the comparison of the water cut curves demonstrated almost the same level of accuracy obtained by $q$-AQGT* and ALG. In other words, for locally measured quantities estimated at the production grid block, $q$-AQGT* models produce the same solution quality as of the iterative procedure of ALG method. The channelized flow patterns are effectively captured from injection to production points by a flow-adapted grid. However, this has not been exactly the case for globally measured error norms shown in Tables 1 and 2. This can be attributed to a slight deviation in the location of high flow region in the $q$-AQGk* and $q$-AQGT* models in comparison to ALG and the reference model, and the error norm sensitivity to these slight dislocations. The spatial discrepancy, while not undermining the water cut prediction, has increased global error norms. Certainly application of an iterative local-global adjustment technique can improve the results of flow-based quadtree grids, albeit with certain computational expenses.

\subsection{Multiphase Simulations}

In order to assess the quality of solutions on adaptive grids in multiphase case, the same simulations with non-linear flow function are performed on layers 37 and 47. In Tables 3 and 4 , the saturation errors are reported. Performing the simulation till 1.2 PVI, similar to the tracer flow case, we observe reasonable performance of $q$-AQGT* models, coarsening level 1. The relative error in terms of $\epsilon_{r}(s)$, either for $M=0.1$ or $M=10$ to ALG is in an acceptable range of 0.9 and 1.2. One has to give credit to updating the upscaled transmissibilities and consequent regard for taking into account the total mobility changes. Other observations are similar to the tracer flow simulation, that is the low performance of $k$-AQGk* and general improvements by transmissibility upscaling for both $k$-AQG and $q$-AQG models.

Figures 8 and 9 show water cut curves obtained by different models (at coarsening level 2) for multiphase case for layers 37 and 47, respectively.

For the permeability upscaling AQG models, the general observation is that, for $M=10$ case, $q$-AQGk* stands in the middle of the ALG curves and RM, $k$-AQGk* curves. The accuracy is not as of the tracer flow case, however, the breakthrough time has been reasonably matched. On the contrary, for the case of $M=0.1$, the breakthrough time is not predicted as of the fine scale reference solution and ALG method by $q$-AQGk*. This can be attributed to the sharp increase of saturation within a small spatial distance due to piston-like movement of the front. In such case, the water cut curves are more sensitive to saturation at the production 
Table 3 The saturation error for layer 37 multiphase flow simulation

\begin{tabular}{lllll}
\hline Model/Method & $\delta(s)_{M=0.1}$ & $\epsilon_{r}(s)_{M=0.1}$ & $\delta(s)_{M=10}$ & $\epsilon_{r}(s)_{M=10}$ \\
\hline RM 1024 c & 0.2797 & 1.7953 & 0.2118 & 2.0095 \\
RM 256 c & 0.3585 & 1.4444 & 0.3023 & 1.5885 \\
ALG 1024 c & 0.1558 & - & 0.1054 & - \\
ALG 256 c & 0.2482 & - & 0.1903 & - \\
$q$-AQGk* 847 c & 0.2908 & 1.5436 & 0.2196 & 1.7234 \\
$q$-AQGk* 403 c & 0.3381 & 2.1442 & 0.2760 & 2.2832 \\
$k$-AQGk* 1066 c & 0.2932 & 1.9591 & 0.2374 & 2.3448 \\
$k$-AQGk* 508 c & 0.3200 & 2.5584 & 0.2717 & 2.8332 \\
$q$-AQGT* 847 c & 0.2149 & 1.1409 & 0.1545 & 1.2125 \\
$q$-AQGT* 403 c & 0.2372 & 1.5045 & 0.1832 & 1.5155 \\
$k$-AQGT* 1066 c & 0.2190 & 1.4633 & 0.1769 & 1.7472 \\
$k$-AQGT* 508 c & 0.2255 & 1.8029 & 0.1890 & 1.9708 \\
\hline
\end{tabular}

Table 4 The saturation error for layer 47 multiphase flow simulation

\begin{tabular}{lllll}
\hline Model/Method & $\delta(s)_{M=0.1}$ & $\epsilon_{r}(s)_{M=0.1}$ & $\delta(s)_{M=10}$ & $\epsilon_{r}(s)_{M=10}$ \\
\hline RM 1024 c & 0.2867 & 1.6143 & 0.3163 & 2.6535 \\
RM 256 c & 0.4346 & 1.5373 & 0.4350 & 1.9308 \\
ALG 1024 c & 0.1776 & - & 0.1192 & - \\
ALG 256 c & 0.2827 & - & 0.2253 & - \\
$q$-AQGk* 640 c & 0.3724 & 1.3105 & 0.3480 & 1.8247 \\
$q$-AQGk* 304 c & 0.4097 & 1.7210 & 0.4189 & 2.2079 \\
$k$-AQGk* 1363 c & 0.3117 & 2.3361 & 0.3639 & 4.0635 \\
$k$-AQGk* 583 c & 0.3815 & 3.0732 & 0.4102 & 4.1463 \\
$q$-AQGT* 640 c & 0.2498 & 0.8791 & 0.2074 & 1.0875 \\
$q$-AQGT* 304 c & 0.2718 & 1.1417 & 0.2164 & 1.1406 \\
$k$-AQGT* 1363 c & 0.1953 & 1.4637 & 0.1430 & 1.5968 \\
$k$-AQGT* 583 c & 0.2599 & 2.0937 & 0.2350 & 2.3754 \\
\hline
\end{tabular}

cell. Such sensitivity is captured effectively by ALG method but not as much effectively by $q$-AQGk* models. For $M=10$, the physical dispersion and gradual increase of saturation and water cut at production cell, have provided a suitable condition for $q$-AQGk* model. The $k$-AQGk* model results in relatively poor quality solutions similar to RM.

For the transmissibility upscaling AQG models, we observe very reasonable performance by $q$-AQGT* compared to ALG in predicting breakthrough time and water cut at simulation end time. $k$-AQGT* also, in its turn, has increased accuracy.

It is also interesting to compare the methods for a different boundary condition. Here we prescribe layers 10,37 and 47 with a constant pressure gradient. That is, we put $p_{i}=100 \mathrm{~atm}$ and $p_{o}=1$ atm at $x=0$ and $x=L$, then we measure the outflow from $x=L$ denoted by $Q_{\mathrm{o}}\left(\mathrm{m}^{3} / \mathrm{s}\right)$. Also we add the uniform grid with extended-local transmissibility upscaling (denoted by Ex $\mathbf{T}^{*}$ ) to the list of compared methods. This method corresponds to ALG on 

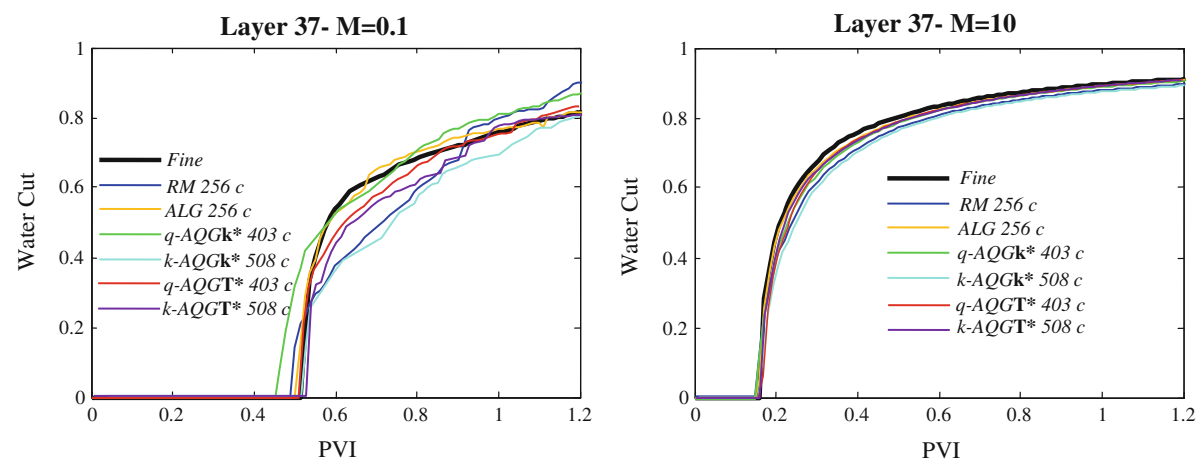

Fig. 8 Comparisons of water cut curves measured at production cell by different models for layer 37 , coarsening level 2, for multiphase simulation
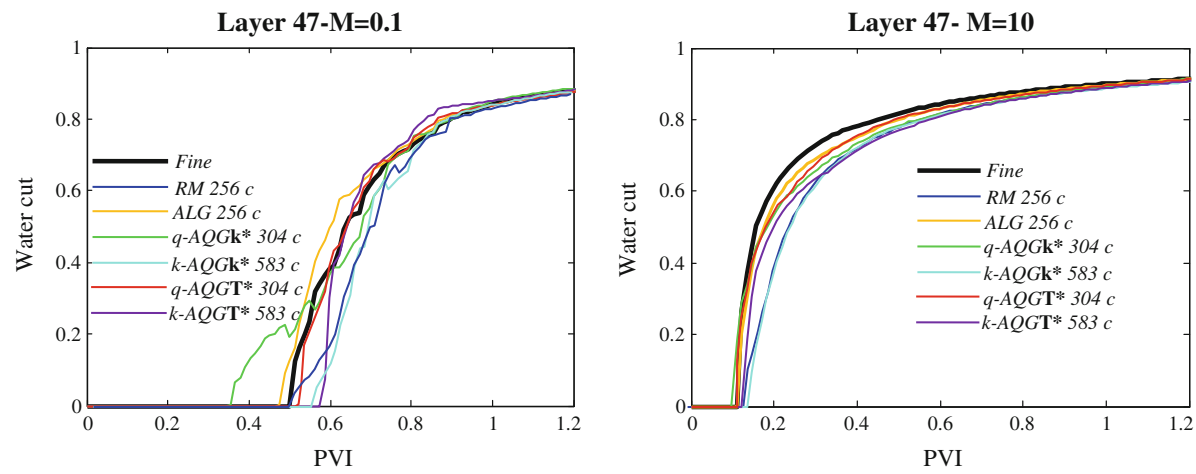

Fig. 9 Comparisons of water cut curves measured at production cell by different models for layer 47, coarsening level 2, for multiphase simulation

uniform grid without iteration. The comparison of outflows obtained by six methods for tracer flow and multiphase flow $(M=10)$ are shown in Fig. 10.

We notice that the permeability upscaling for RM and $q$-AQGk* has led to significant underestimation of flow for layers 37 and 47 . Also the error for this type of boundary condition is more pronounced than source and sink pattern because the error includes aggregation of errors for entire cells next to the boundary. It is clear from comparison that, for this special case of boundary condition, the methods are generally ranked from high performance to low performance as: RM, $q$-AQGk*,Ex $\mathbf{T}^{*}, q$-AQGT* and ALG. This illustrates that local-global upscaling, adaptive gridding and extended-local transmissibility upscaling, on their turns, all improve the quality of upscaling.

\subsection{Presence of Shale in Porous Media}

In the previous sections, we studied several subsurface flow problems representing fluvial channels and high permeability flow-paths that create problems for upscaling. In this section, we study problems where flow barriers in the form of shale with very low permeability is present. These flow barriers can also be problematic for upscaling. For example, renormalization upscaling treats shales imprecisely and results in a deformation of shales which can 

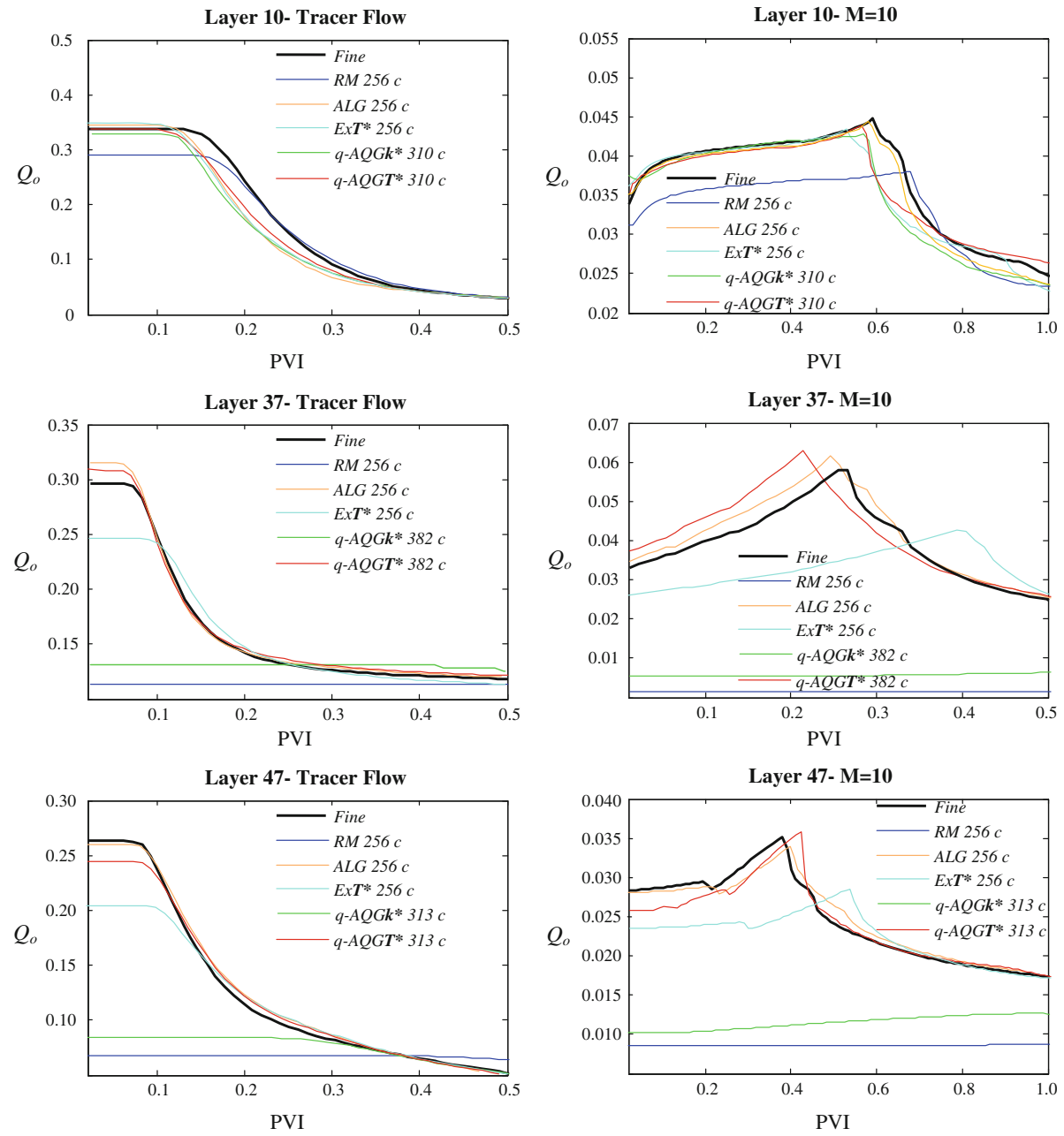

Fig. 10 The comparison of outflow for constant pressure gradient case

lead to misjudgement of the reservoir connectivity. Typically, shales have a large aspect ratio and they can be distributed against the flow direction.

Moreover, the presence of flow barriers might be problematic for velocity reconstruction. Aarnes et al. (2006), for example, have shown that for a barrier that is stretched beyond a coarse scale grid block, the boundary conditions interpolated from coarse velocities in multiscale method represent erroneous assumptions that may produce an unnatural amount of flow through the barrier. That is, the barrier is totally or partially ignored by the reconstructed velocity field. In the downscaling algorithm used here, also a particular position of shale is a source of error: the shale is stretching out of the coarse grid boundaries so that tail or head of the shale is not situated either inside the coarse grid or over the boundaries.

In this section we examine a medium consisting of both channelized heterogeneities and few flow barriers. Figure 11a shows the fine scale permeability field used. This field is an overlay of few flow barriers on the layer 37 of the SPE10 model. These barriers are cutting the 


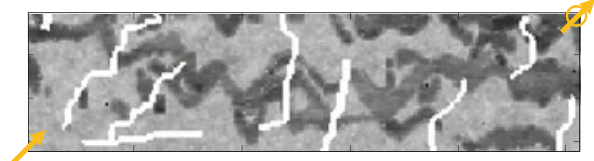

(a)

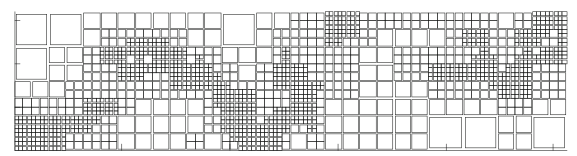

(c)

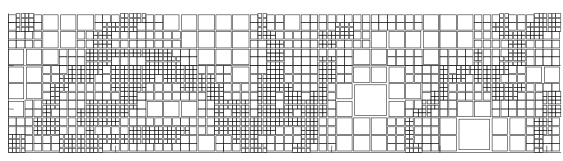

(e)

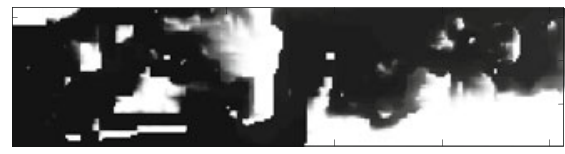

(g)

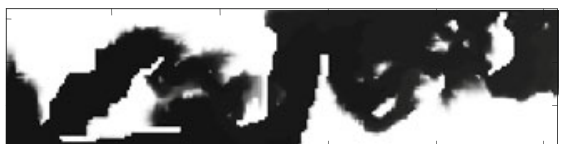

(b)

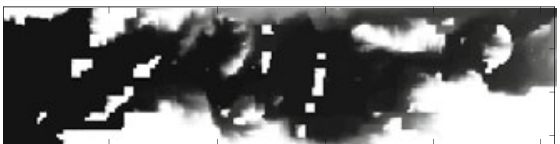

(d)

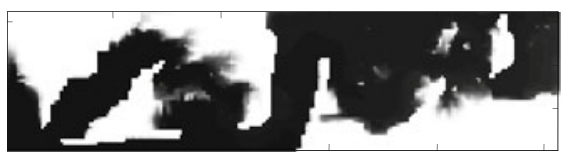

(f)

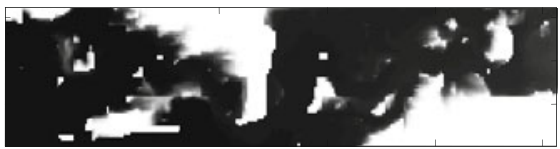

(h)

Fig. 11 Tracer flow simulation on layer 37 combined with few streaks of shale. a The permeability field, b the saturation profile from the reference fine model at $t=1.2 \mathrm{PVI}$, black is water while white is oil, $\mathbf{c}$ the $q$-AQG model with 1408 cells, $\mathbf{d}$ the saturation profile at 1.2 PVI from $q$-AQGk* downscaled velocities which is very similar to $q$-AQGT* for the tracer flow, $\mathbf{e}$ the $q h$-AQG model with 1678 cells, $\mathbf{f}$ the saturation profile at 1.2 PVI from $q h$-AQGk* downscaled velocities which is very similar to $q h$-AQGT*, $\mathbf{g}$ the saturation profile at 1.2 PVI from RM downscaled velocities, and $\mathbf{h}$ the saturation profile at 1.2 PVI from ALG downscaled velocities

high flow regions of the model. Figure $11 \mathrm{~b}$ shows the saturation profile at $1.2 \mathrm{PVI}$ obtained by solving the fine scale problem for the tracer flow simulation. The refinement criterion used in $q$-AQG is modified to refine the grid at zero permeabilities as well as high flow areas. The new refinement heuristic is denoted by $q h$-AQG. The grid for $q$-AQG is shown in Fig. 11c with 1408 cells. The resultant grid for $q h$-AQG is shown in Fig. 11e with 1678 cells, refining both the areas containing barriers and inducing high flow.

The resultant saturation profile from downscaling of tracer flow simulation on these grids with permeability upscaling are shown, respectively, in Fig. 11d, f. For RM 1024 cells and ALG 1024 cells, the obtained saturation profiles after velocity downscaling are shown in Fig. $11 \mathrm{~g}$, h, respectively. At $t=1.2 \mathrm{PVI}$, the value of $\delta(s)$ for RM 1024 cells, ALG 1024 cells, $q$-AQG 1408 cells and qh-AQG 1678 cells is $0.5827,0.5008,0.5196$ and 0.2028 , respectively. These numbers shows significantly small errors for $q h-\mathrm{AQG}$ in comparison to other methods.

We plotted water cut curves obtained by these models at coarsening level 1 in Fig. 12. The water cut curves demonstrate that either of $q h$-AQGk* and $q h$-AQGT* models produce considerably more accurate result in comparison to RM, ALG, $q$-AQGk* and $q$-AQGT*.

Now being assured by the new scheme of quadtree grid generation, we examine the model with multiphase flow with viscosity ratios as before. We only assess the models at the coarsening level 1 . The water cut curves obtained by $M=0.1$ and $M=10$ are shown in Fig. 13 at $t=1.2$ PVI. These results shows that $q h$-AQGT* for multiphase flow especially for the case of $M=0.1$ is as effective as in the case of tracer flow simulation. 
Fig. 12 The water cut curves for layer 37 with shale at coarsening level 1 , tracer flow simulation
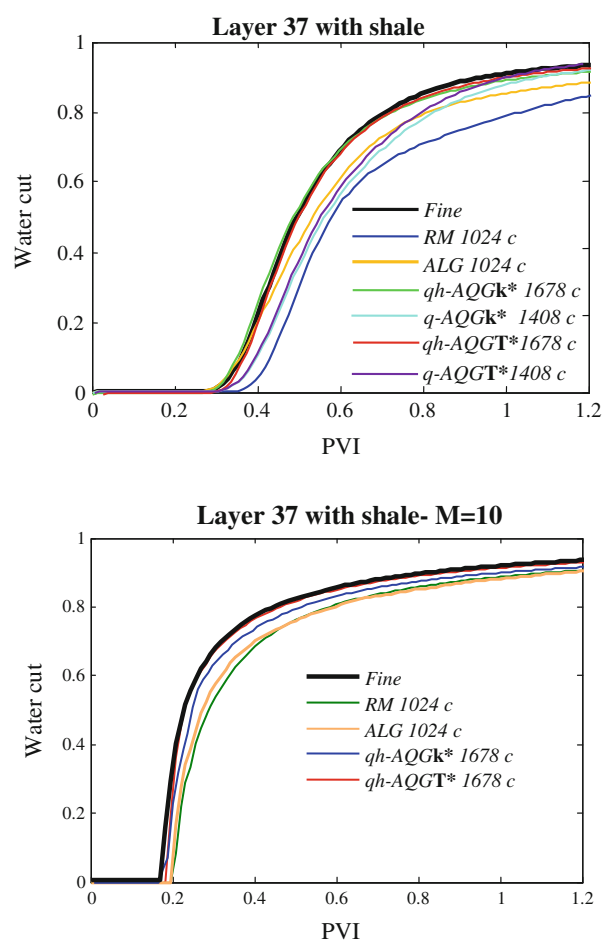

Fig. 13 The water cut curves for layer 37 with shale at two viscosity ratios, multiphase flow simulation

\section{Conclusions}

We have presented a critical evaluation of different methods of quadtree construction. These methods were compared to uniform grid upscaling. For the quadtree design, two adaptivity criteria were considered: flow- and permeability-based. For the uniform grid, renormalization and local-global upscaling were used. Based on the extensive numerical testing on single phase and multiphase flow simulations the following conclusions are drawn:

1. A permeability-based AQG coupled with renormalization permeability upscaling ( $k$-AQGk*) led to significant errors in tracer flow simulations of channelized systems. This can be attributed to either the loss of channels continuity or to an inaccurate grid refinement.

2. A flow-based AQG coupled with renormalization permeability upscaling ( $q$-AQGk*) and extended-local transmissibility upscaling ( $q$-AQGT*) showed good results compared to adaptive local-global (ALG) upscaling for the production outputs (water cut curves) in the tracer flow test cases.

3. For the case of multiphase simulations, $q$-AQGT* shows consistent good performance, however, the quality of $q$-AQGk* water cut curves depended on the viscosities of engaging fluids. That is, for high values of viscosity ratios (ratio of viscosity of displaced fluid over viscosity of displacing one) we have a satisfying agreement between $q$-AQGk* and the fine grid model for the production outputs. For low values of viscosity ratio, the production data obtained by $q$-AQGk* shows some deviation from the reference results. For high viscosity ratios, this can be largely attributed to the predominantly streaming flow 
through the channels and streaks that the adaptive grid has refined around. In contrast, for the case of low viscosity ratios, the flow is piston-like, hence more sweep-efficient, and not necessarily dispersing through the channels.

4. Finally, we inserted streaks of shale as forms of barriers inside a test reservoir. For this pattern, $q$-AQGT* model was tuned heuristically for refining around the shale in addition to the flow-based refinement indicator. For the tracer flow and multiphase case, the results in terms of water production values and saturation profiles were comparable to the fine scale solution and with higher number of grid cells, it easily outperformed ALG in quality and production prediction.

Quadtrees can be dynamically adapted during the simulation time. This might result in a robust method for dynamic upscaling for multiphase flow problems. Testing dynamically adapted flow-based quadtree grids combined with downscaling can be a promising line of future research.

As we mentioned in the text, comparison of flow-based refinement indicator used by our quadtree gridding ( $q$-AQGT*) with that of Cartesian cell-based anisotropic refinement by Gerritsen and Lambers (2008) remains an unfinished task. This would be interesting to investigate whether anisotropic refinement and multilevel local-global upscaling could increase the accuracy sufficiently so that extra computations would be justified. This has been the case for Cartesian cell-based anisotropic refinement by Gerritsen and Lambers (2008).

Acknowledgments The authors are grateful to one of the anonymous reviewers for suggesting to implement transmissibility-based upscaling. This suggestion has made the numerical comparison more consistent. We also, would like to thank the editor and the anonymous reviewers for their valuable comments and suggestions to improve the quality of the paper.

\section{References}

Aarnes, J.E., Efendiev, Y.: An adaptive multiscale method for simulation of fluid flow in heterogeneous porous media. Multiscale Model. Simul. 5(3), 918-939 (2006)

Aarnes, J.E., Krogstad, S., Lie, K.A.: A hierarchical multiscale method for two-phase flow based upon mixed finite elements and nonuniform coarse grids. Multiscale Model. Simul. 5(2), 337-363 (2006)

Babaei, M., King, P.R.: A modified nested-gridding for upscaling downscaling in reservoir simulation. Transp. Porous Med. 93(3), 753-775 (2012)

Batycky, R.P., Blunt, M.J., Thiele, M.R.: A 3D field-scale streamline-based reservoir simulator. SPE Reserv. Eng. 12(4), 246-254 (1997)

Begg, S., Carter, R., Dranfield, P.: Assigning effective values to simulator gridblock parameters for heterogeneous reservoirs. SPE Reserv. Eng. 4(4), 455-463 (1989)

Castellini, A.: Flow based grids for reservoir simulation. Stanford University, Stanford, CA, Master's thesis (2001)

Castellini, A., Edwards, M.G., Durlofsky, L.J.: Flow based modules for grid generation in two and three dimensions. In: Proceedings of 7th European Conference on the Mathematics of Oil Recovery, Baveno, Italy (2000).

Chen, Y., Durlofsky, L.J.: Adaptive local-global upscaling for general flow scenarios in heterogeneous formations. Transp. Porous Med. 62(2), 157-185 (2006a)

Chen, Y., Durlofsky, L.J.: Efficient incorporation of global effects in upscaled models of two-phase flow and transport in heterogeneous formations. Multiscale Model. Simul. 5, 445-475 (2006b)

Chen, Y., Li, Y.: Local-global two-phase upscaling of flow and transport in heterogeneous formations. Multiscale Model. Simul. 8, 125-153 (2009)

Chen, Y., Durlofsky, L., Gerritsen, M., Wen, X.H.: A coupled local-global upscaling approach for simulating flow in highly heterogeneous formations. Adv. Water Resour. 26(10), 1041-1060 (2003)

Christie, M.: Upscaling for reservoir simulation. J. Petroleum Technol. 48(11), 1004-1010 (1996) 
Christie, M., Blunt, M.: Tenth SPE comparative solution project: a comparison of upscaling techniques. SPE Reserv. Eval. Eng. 4(4), 308-317 (2001)

Corey, A.: The interrelation between gas and oil relative permeabilities. Producers Mon. 19(1), 38-41 (1954)

Darman, N., Durlofsky, L.J., Sorbie, K., Pickup, G.: Upscaling immiscible gas displacements: quantitative use of fine-grid flow data in grid-coarsening schemes. SPE J. 6(1), 47-56 (2001)

de Berg, M., van Kreveld, M., Overmars, M., Schwarzkopf, O.: Computational Geometry: Algorithms and Applications. Springer, Berlin (2000)

Donoho, D.L.: De-noising by soft-thresholding. IEEE Trans. Inf. Theory 41(3), 613-627 (1995)

Donoho, D.L., Johnstone, I.M.: Adapting to unknown smoothness via wavelet shrinkage. J. Am. Stat. Assoc. 90, 1200-1224 (1995)

Durlofsky, L.J.: Numerical calculation of equivalent grid block permeability tensors for heterogeneous porous media. Water Resour. Res. 27(5), 699-708 (1991)

Durlofsky, L.J.: Use of higher moments for the description of upscaled, process independent relative permeabilities. SPE J. 2(4), 474-484 (1997)

Durlofsky, L.J.: Upscaling and gridding of fine scale geological models for flow simulation. In: Proceedings of 8th International Forum on Reservoir Simulation, Iles Borromees, Stresa, Italy (2005).

Durlofsky, L.J., Behrens, R., Jones, R., Bernath, A.: Scale up of heterogeneous three dimensional reservoir descriptions. SPE J. 1(3), 313-326 (1996)

Durlofsky, L.J., Jones, R.C., Milliken, W.J.: A nonuniform coarsening approach for the scale-up of displacement processes in heterogeneous porous media. Adv. Water Resour. 20(5-6), 335-347 (1997)

Ebrahimi, F., Sahimi, M.: Multiresolution wavelet coarsening and analysis of transport in heterogeneous media. Physica A 316(1-4), 160-188 (2002)

Ebrahimi, F., Sahimi, M.: Grid coarsening, simulation of transport processes in, and scale-up of heterogeneous media: application of multiresolution wavelet transformations. Mech. Mater. 38(8-10), 772-785 (2004)

Edwards, M.G.: Elimination of adaptive grid interface errors in the discrete cell centered pressure equation. J. Comput. Phys. 126(2), 356-372 (1996)

Edwards, M.G.: Unstructured, control-volume distributed, full-tensor finite-volume schemes with flow based grids. Comput. Geosci. 6(3), 433-452 (2002)

Efendiev, Y., Durlofsky, L.J.: A generalized convection-diffusion model for subgrid transport in porous media. Multiscale Model. Simul. 1, 504-526 (2003)

Farmer, C., Heath, D., Moody, R.: A global optimization approach to grid generation. Proceedings of SPE Symposium on Reservoir Simulation, Anaheim, California, In (1991)

Forsyth, P., Sammon, P.: Local mesh refinement and modeling of faults and pinchouts. SPE Form. Eval. 1(3), 275-285 (1986)

Garcia, M., Journel, A., Aziz, K.: Automatic grid generation for modeling reservoir heterogeneities. SPE Reserv. Eng. 7(2), 278-284 (1992)

Gerritsen, M., Lambers, J.V.: Integration of local-global upscaling and grid adaptivity for simulation of subsurface flow in heterogeneous formations. Comput. Geosci. 12(2), 193-208 (2008)

Hauge, V.L., Lie, K.A., Natvig, J.R.: Flow-based coarsening for multiscale simulation of transport in porous media. Comput. Geosci. 16(2), 391-408 (2011)

$\mathrm{He}, \mathrm{C} .:$ Structured flow-based gridding and upscaling for reservoir simulation. $\mathrm{PhD}$ thesis, Stanford University (2005).

He, C., Durlofsky, L.J.: Structured flow-based gridding and upscaling for modeling subsurface flow. Adv. Water Resour. 29(12), 1876-1892 (2006)

King, P.R.: The use of renormalization for calculating effective permeability. Transp. Porous Med. 4(1), 37-58 (1989)

Kippe, V., Aarnes, J.E., Lie, K.A.: A comparison of multiscale methods for elliptic problems in porous media flow. Comput. Geosci. 12(3), 377-398 (2008)

Lee, S.H., Zhou, H., Tchelepi, H.A.: Adaptive multiscale finite-volume method for nonlinear multiphase transport in heterogeneous formations. J. Comput. Phys. 228(24), 9036-9058 (2009)

Li, D., Cullick, A.S., Lake, L.W.: Global scale-up of reservoir model permeability with local grid refinement. J. Petroleum Sci. Eng. 14(1-2), 1-13 (1995)

Mlacnik, M., Durlofsky, L.J., Heinemann, Z.: Sequentially adapted flow-based PEBI grids for reservoir simulation. SPE J. 11(3), 317-327 (2006)

Nilsson, J., Gerritsen, M., Younis, R.: A novel adaptive anisotropic grid framework for efficient reservoir simulation. Proceedings of SPE Reservoir Simulation Symposium, The Woodlands, Texas, In (2005)

Pickup, G.E., Ringrose, P.S., Jensen, J.L., Sorbie, K.S.: Permeability tensors for sedimentary structures. Math. Geol. 26(2), 227-250 (1994)

Prevost, M., Lepage, F., Durlofsky, L.J., Mallet, J.L.: Unstructured 3D gridding and upscaling for coarse modelling of geometrically complex reservoirs. Petroleum Geosci. 11(4), 339-345 (2005) 
Qi, D., Wong, P., Liu, K.: An improved global upscaling approach for reservoir simulation. Petroleum Sci. Technol. 19(7-8), 779-795 (2001)

Quandalle, P., Besset, P.: Reduction of grid effects due to local sub-gridding in simulations using a composite grid. Proceedings of SPE Reservoir Simulation Symposium, Dallas, Texas, In (1985)

Rasaei, M.R., Sahimi, M.: Upscaling and simulation of waterflooding in heterogeneous reservoirs using wavelet transformations: application to the SPE-10 model. Transp. Porous Med. 72(3), 311-338 (2008a)

Rasaei, M.R., Sahimi, M.: Upscaling of the permeability by multiscale wavelet transformations and simulation of multiphase flows in heterogeneous porous media. Comput. Geosci. 13(2), 187-214 (2008b)

Stein, C.M.: Estimation of the mean of a multivariate normal distribution. Ann. Stat. 9(6), 1135-1151 (1981)

Wen, X.H., Gómez-Hernández, J.J.: Upscaling hydraulic conductivities in heterogeneous media: an overview. J. Hydrol. 183(1-2):ix-xxxii (1996)

Wen, X.H., Durlofsky, L.J., Edwards, M.G.: Upscaling of channel systems in two dimensions using flow-based grids. Transp. Porous Med. 51(3), 343-366 (2003)

Younis, R., Caers, J.: A method for static-based upgridding. In: Proceedings of the 8th European Conference on the Mathematics of Oil Recovery (2002) 